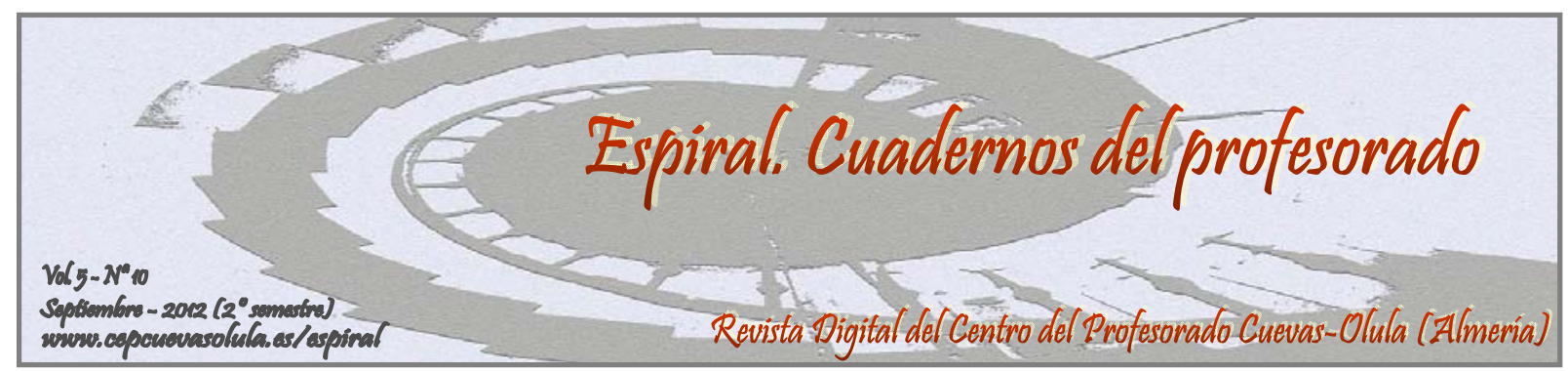

\title{
OPUS SOLIDARIDAD: TRÍO PARA MÚSICA, EDUCACIÓN Y VALORES
}

\author{
OPUS SOLIDARITY: MUSIC-EDUCATION-VALUES TRIO \\ Cecilia María Azorín Abellán ${ }^{(1)}$ y Gregorio Vicente Nicolás ${ }^{(2)}$
}

(1) Maestra de Música de Educación Primaria

(2) Departamento de Expresión Plástica, Musical y Dinámica, Facultad de Educación, Universidad de Murcia, España

RESUMEN: El objetivo de esta experiencia de innovación es diseñar y aplicar una propuesta didáctica de educación en valores, concretamente, el valor de la solidaridad, contextualizada en el ámbito de la educación musical. El diseño metodológico de esta investigación es cuasi-experimental de grupo único. Se pretende observar la respuesta de los discentes ante una propuesta didáctica basada en el valor de la solidaridad, trabajada en un marco temporal determinado con unas actividades específicas. Se ha implementado en $5^{\circ}$ curso de educación primaria en un centro de la Región de Murcia, con duración total de siete sesiones. Para la evaluación inicial y sumativa de la propuesta se han creado dos cuestionarios, uno inicial (ideas previas) y otro final o de evaluación. Los resultados obtenidos revelan que los discentes han acogido la experiencia con gran entusiasmo, mostrando interés por las actividades presentadas, participando activamente en ellas. Así mismo, se ha constatado una aproximación del alumnado al concepto de solidaridad.

Palabras clave: música, educación en valores, Educación Primaria, solidaridad

ABSTRACT: The objective of this innovation experience is to design and implement a didactic education in values, specifically, the value of solidarity, contextualized in the field of music education. The methodological design of this research is quasi-experimental single group. The aim is to observe the response of the learners to an educational proposal based on the value of solidarity. The experience has been implemented in year 5 of Primary Education in a school of Murcia. It lasted seven lessons. For the initial and summative assessment of the unit we have developed two questionnaires: one initial (preconceptions) and a final report. Results show that learners have embraced the experience with great enthusiasm, showing interested in the activities presented by actively participating by the children. Also, it has found a student's approach to the concept of solidarity.

Key words: music, values education, Primary Education, solidarity

Azorín Abellán, C.M. y Vicente Nicolás, G. (2012). Opus solidaridad: trío para música, educación y valores. Espiral. Cuadernos del Profesorado, 5(10), 78-86. Disponible en: http://www.cepcuevasolula.es/espiral.

Fecha de recepción: 31/01/2012

Fecha de aceptación: 06/06/2012
Enviar correspondencia a: gvicente@um.es 


\section{1.- INTRODUCCIÓN}

Vivimos en un mundo individualista, nos movemos por regímenes de concurrencia competitiva, nos preocupamos sólo de aquello que acontece a nuestro alrededor... En este contexto surge un concepto de sociedad sin valores, crisis de valores y emulsión de nuevos valores fusionados con otros ya existentes (Touriñán, 2009).

En el ámbito educativo, la praxis diaria en el aula desvela en ocasiones una ausencia de disciplina, amabilidad, respeto, sinceridad, gratitud y un exceso de agresividad, individualismo e insolidaridad. Ante la necesidad de una respuesta por parte de la comunidad educativa, Peñalver (2010) considera muy pertinente la introducción en el currículo de una enseñanza que tenga presente la formación en valores y sea una respuesta a esta problemática social. Igualmente, Herrera (2007) insiste en la necesidad de la inclusión de la educación en valores en los currículos educativos.

La etapa de Educación Primaria es uno de los momentos en que el alumnado se desarrolla de forma plena e integral, construye su personalidad e interioriza los valores que guiarán su actuación futura en la sociedad. Así mismo, los continuos cambios emergentes en los comportamientos y valores de la misma, impulsan al profesorado a programar actividades cuyo objetivo es formar a futuros ciudadanos comprometidos que reflexionen sobre la importancia de la solidaridad en sus vidas.

En contraposición al panorama tan desalentador mencionado con anterioridad, la solidaridad se presenta como un valor contrario al individualismo y al egoísmo, que se refleja en el servicio y busca del bien común, su finalidad es solucionar las carencias de los demás, requiere ponerse en el lugar del otro, empatía. La solidaridad es un valor que se aprende, no nacemos personas solidarias. La educación desempeña un rol fundamental en la aprehensión de dicho valor. Como señala Vicente (2007), el punto de partida en este aprendizaje será la experiencia y el acercamiento a personas que muestran conductas solidarias.

La experiencia de innovación docente que presentamos en este artículo con el título de Opus Solidaridad: trío para música, educación y valores, surge de la necesidad de elaborar un material didáctico que trabaje el valor de la solidaridad en el aula de música de una forma integral, lúdica y motivadora, y que sea una repuesta a las necesidades e intereses del alumnado.

La finalidad de nuestro trabajo ha sido reflexionar sobre la importancia de la solidaridad y la modificación o cambio de conducta por parte de nuestros discentes, otorgándoles una serie de pautas y experiencias que favorezcan la aproximación a dicho valor a través de las actividades musicales elaboradas. El proyecto se ha llevado a cabo en el C.E.I.P. Los Álamos de Murcia con el alumnado de $5^{\circ}$ curso y con una duración de 4 semanas ( 7 sesiones) durante el segundo trimestre del curso escolar 2010-2011.

\section{2.- DISEÑO DE LA PROPUESTA EDUCATIVA}

Además de los objetivos estrictamente musicales propios de cualquier unidad didáctica de educación musical, hemos incluido los siguientes de tipo axiológico:

- Favorecer la aproximación del alumnado a acciones y actitudes solidarias que le permitan la aprehensión del significado del valor de la solidaridad.

- Adquirir actitudes solidarias a través de la realización de actividades de movimiento, canto, instrumentación y audición.

- Analizar el texto de canciones relacionadas con la solidaridad con una actitud críticoreflexiva.

- Expresar y comunicar con autonomía e iniciativa personal propuestas solidarias para llevar a cabo en el aula de música.

- Conocer ejemplos actuales de situaciones solidarias que han llevado a cabo con implicación de la actividad musical. 
Los contenidos musicales trabajados habitualmente en clase se han abordado desde la perspectiva de la educación en valores y el concepto de solidaridad. De esta forma se han abarcado contenidos como:

- La escucha, comentario y análisis de músicas y canciones de temática relacionada con la solidaridad.

- Interpretación vocal e instrumental de obras que versan sobre dicho valor.

- Valoración de la importancia de la solidaridad en el mundo a través de la música, en general, y elaboración de propuestas solidarias para el aula de música, en particular.

- Participación en actividades grupales respetando las opiniones y aportaciones de los demás.

La metodología utilizada ha partido de los conocimientos previos que el alumnado posee y su propia experiencia. Se ha prestado especial importancia al carácter lúdico de las actividades para favorecer la motivación. Igualmente, el rol del profesorado constituye un eje central metodológico y debe ser un referente de solidaridad a través de la actitud que mantiene hacia el alumnado y la forma de plantear las actividades y de resolver conflictos. Para que muchas de las actividades diseñadas adquieran una verdadera dimensión axiológica, es necesario incluir en algún momento de su desarrollo la posibilidad de opinar, expresar, dialogar o debatir.

Para la evaluación de la unidad didáctica se ha diseñado un cuestionario inicial de ideas previas sobre la solidaridad y un cuestionario final para constatar el grado de adquisición de los conceptos e ideas trabajadas. La literatura revisada nos revela que son poco frecuentes las investigaciones que abordan el valor de la solidaridad desde un enfoque educativo-musical y mucho más escasas aquellas que utilizan la técnica del cuestionario. Para la realización de nuestros cuestionarios se han tenido como referentes otros que, si bien no versan sobre la temática música y solidaridad, afrontan parcialmente estos tópicos. Entre ellos podemos citar los elaborados por Tiburcio (2010) o el Test de la Solidaridad, perteneciente a la web Gente Solidaria ${ }^{1}$ del CEIP San José de Calasanz (Valencia), creado por el profesor Martín Cetina en 2010. Dicho Test ha inspirado algunas de las preguntas contenidas en nuestros instrumentos de evaluación.

Mediante el cuestionario inicial se ha pretendido conocer las ideas previas del alumnado sobre el concepto solidaridad y actitudes relacionadas con dicho valor. Debido al carácter inmaterial de este concepto, se han formulado preguntas y supuestos que hacen referencia a situaciones de la vida cotidiana del alumnado, que permitan la evidencia o materialización del valor de la solidaridad. Con respecto al cuestionario final, sus objetivos han sido: (1) constatar los conocimientos adquiridos a lo largo de la unidad didáctica, (2) conocer el grado de adquisición del concepto solidaridad y su relación con el aula de música, (3) comprobar si han adquirido pautas de actuación útiles para su vida cotidiana y (4) conocer su opinión sobre las actividades realizadas.

Además, se han utilizado otras técnicas de recogida de datos como la observación directa, mediante registros anecdóticos; la grabación en vídeo, que posteriormente se ha presentado al alumnado para favorecer la autoevaluación; y, por último, las producciones de los escolares.

\section{3.- DISEÑO DE LAS ACTIVIDADES}

Para el diseño de las actividades hemos tomado como referencia el itinerario propuesto por Ortega y Mínguez (2001) para la educación en la solidaridad. Dicho itinerario define los siguientes ámbitos de trabajo: (1) toma de conciencia de las situaciones de marginación y pobreza de individuos y pueblos, (2) descubrimiento de las causas que producen la marginación y la pobreza, (3) reconocimiento de la dignidad de toda persona que reclama una respuesta, (4) entendimiento del carácter global de los problemas que afectan a la humanidad, (5) encuentro de la solidaridad en el entorno más próximo, y (6) la solidaridad en mi conducta personal, compromiso y formas de actuación. Las actividades diseñadas más destacadas han sido:

\footnotetext{
${ }^{1}$ Puede consultarse en http://calasanz.edu.gva.es/gente-solidaria/test-solidaridad.html
} 
- Disco-fórum sobre la canción Solaridad de Eros Ramazzotti. Tras la primera audición de la canción, el alumnado completa las palabras que se han eliminado de una ficha que contiene el texto de Solaridad, al mismo tiempo que escuchan por segunda vez la canción. Una vez que la melodía y la letra es más familiar para el grupo, se canta a modo de karaoke (disponible en http://www.youtube.com/watch?v=klu96LL222E). Posteriormente, se analiza la letra de la canción atendiendo a aquellos aspectos que hemos resaltado en negrita (ej.: "abrirá los ojos que se están acostumbrando a no querer ver"). También se trabaja el significado de la palabra Solaridad, término que Ramazzotti canta refiriéndose a la solidaridad. Finalmente, se realiza en la pizarra una lluvia de ideas sobre palabras que están relacionadas con la solidaridad.

- A partir de las ideas planteadas en el disco-fórum, se realizan propuestas de solidaridad en el aula de música en formato cartulina para ponerlas en la clase. Un ejemplo de propuesta ha sido: Comprometernos a respetar los materiales del aula de música para que otros compañeros puedan también disfrutar de ellos. Por parejas, elaboramos un total de doce propuestas. Cada pareja decora en cartulina su propuesta y la comenta a todo el grupo (figura 1).

- Lluvia de ideas sonoras con percusión corporal. Retomamos las palabras relacionadas con la soli-

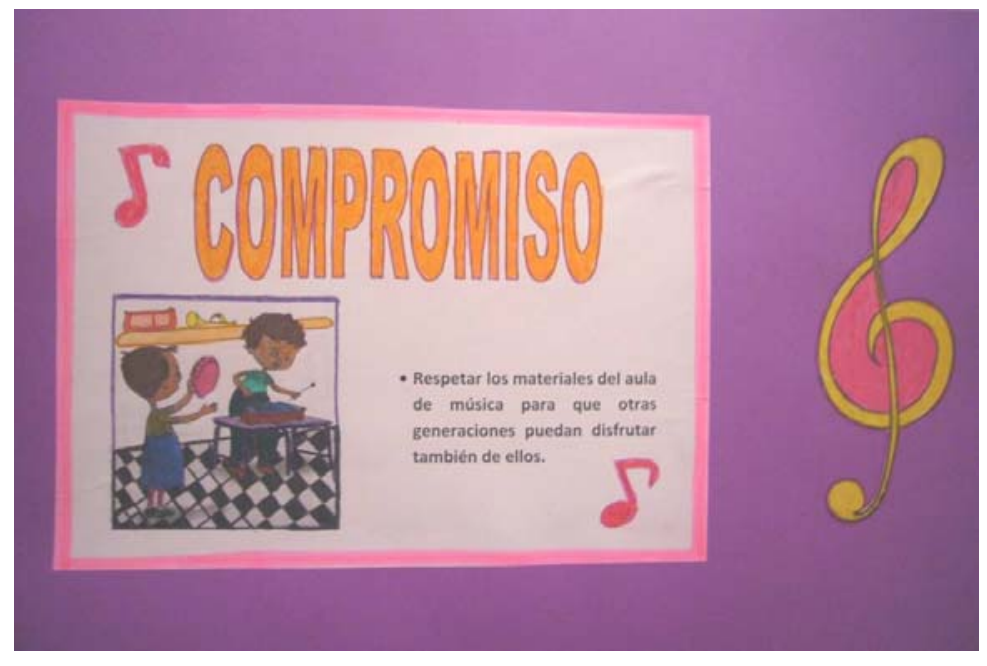

Figura 1. Ejemplo de propuesta solidaria daridad de la clase anterior y a cada una de ellas se le asigna un esquema rítmico-corporal dependiendo del número de sílabas (ej.: Com-pro-mi-so . . . .).

- Audición/vídeo de la canción Chiquitita de Amaia Montero. Comentario y debate de una actividad musical realizada con fines solidarios, en este caso, recaudar fondos para la educación de niños en colaboración con Unicef.

- Visitamos el blog creado para esta Unidad Didáctica llamado Solidaridad en el aula de música (http://solidaridadaulademusica.blogspot.com/) (figura 2). Entregamos al alumnado el documento elaborado para completar las actividades propuestas en el blog. Éste incluye vídeos de actuaciones relacionadas con el valor de la solidaridad ("Ay Haití" e "Imagine", entre otros) y diferentes cuestiones como: ¿qué artistas, cantantes, futbolistas, etc. se implican cantando la canción por una causa solidaria?, ¿cuándo es el Día Internacional de la Solidaridad?, ¿cuándo y dónde tuvo lugar el Concierto Solidario que la Federación de Bandas de Música de la Región de Murcia realizó a favor de una ONG?, ¿qué puedes hacer para ser más solidario y solidaria en el aula de música?

- Instrumentación del Himno de la Alegría. Provocamos un conflicto: no disponemos de instrumentos para todos, ¿qué hacemos?, ¿cómo nos organizamos? Favorecemos el diálogo y el consenso en el alumnado. Finalmente se establecen turnos para tocar los instrumentos. Reflexionamos sobre lo que ha ocurrido en la clase. 


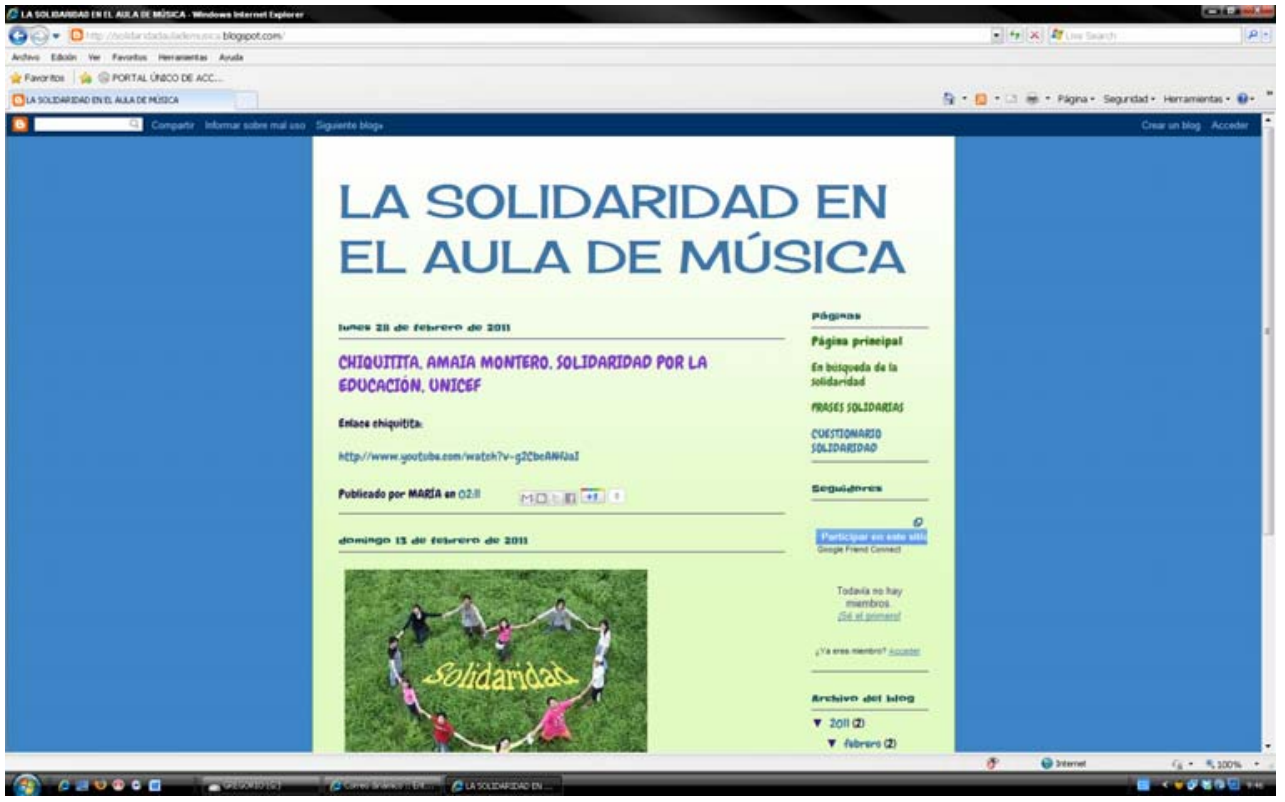

Figura 2. Captura del Blog Solidaridad en el aula de Música

- Canción Solidaridad de Cecilia María Azorín. Leemos y aprendemos la canción creada para esta Unidad Didáctica y, posteriormente, introducimos su coreografía. Cantamos y danzamos en círculo cogidos de las manos. Cada vez que paramos música y volvemos a empezar cambiamos de compañeros. Todos bailamos con todos (figura 3).

\section{SOLIDARIDAD}

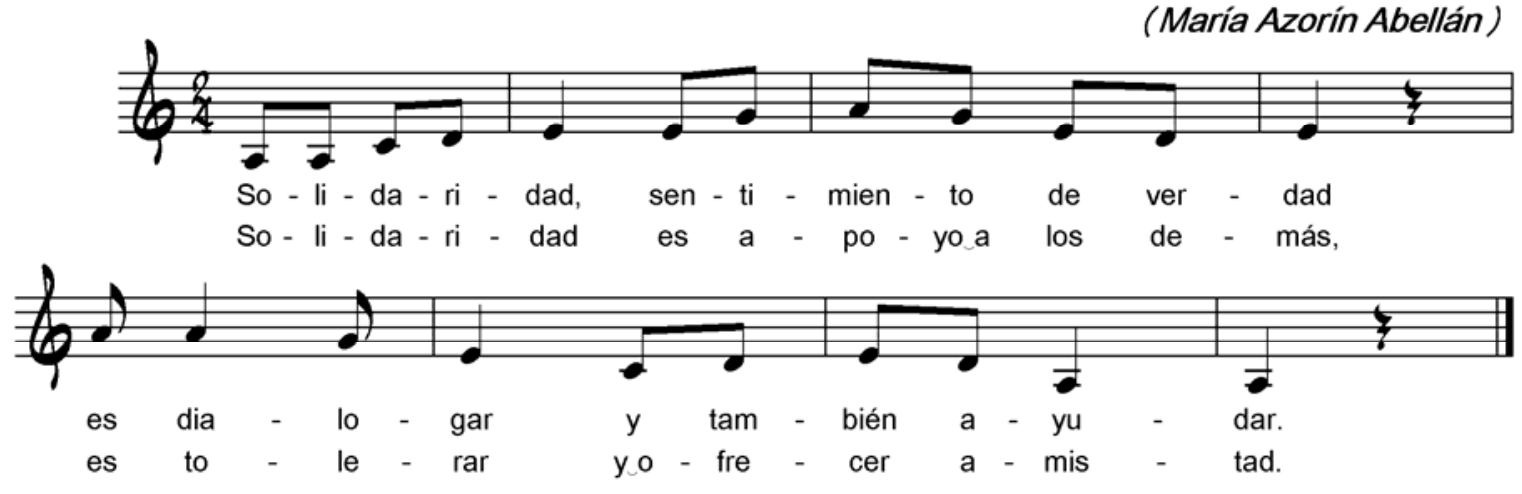

Figura 3. Canción Solidaridad: partitura del alumnado

\section{4.- FASES DE PLANIFICACIÓN DEL TRABAJO}

La experiencia realizada, ha tenido una fase previa (revisión teórica), selección de materiales y establecimiento de los objetivos del trabajo. Una fase de desarrollo (puesta en práctica de la Unidad Didáctica) y fase de evaluación (elaboración del informe final, análisis resultados, conclusiones, etc.). En la tabla 1 se explica de forma más detallada las diferentes fases de desarrollo que ha seguido nuestra propuesta de innovación. 
Tabla 1. Fases de planificación del trabajo

\begin{tabular}{|c|c|c|}
\hline FASE & OBJETIVOS & TAREAS \\
\hline \multirow{3}{*}{ 章 } & \multirow{3}{*}{$\begin{array}{l}\text { - Conocer el estado de la cuestión: ante- } \\
\text { cedentes y estado actual del problema de } \\
\text { investigación (temática: música y valo- } \\
\text { res). }\end{array}$} & - Búsqueda de información. \\
\hline & & - Revisión bibliográfica. \\
\hline & & - Análisis del currículo y legislación educativa. \\
\hline \multirow{3}{*}{ 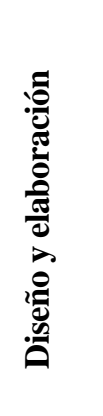 } & \multirow{2}{*}{$\begin{array}{l}\text { - Diseñar una unidad didáctica de } 7 \text { se- } \\
\text { siones para } 5^{\circ} \text { curso de educación prima- } \\
\text { ria que trabaje el valor de la solidaridad } \\
\text { y la música. }\end{array}$} & $\begin{array}{l}\text { - Planificación de objetivos, contenidos, activi- } \\
\text { dades y criterios de evaluación de las sesiones } \\
\text { didácticas. }\end{array}$ \\
\hline & & $\begin{array}{l}\text { - Elaboración de un cuestionario inicial para co- } \\
\text { nocer las ideas previas de los alumnos. }\end{array}$ \\
\hline & $\begin{array}{l}\text { - Elaborar instrumentos de evaluación } \\
\text { de las sesiones. }\end{array}$ & $\begin{array}{l}\text { - Creación de un cuestionario final de conoci- } \\
\text { mientos adquiridos, incluyendo la definición li- } \\
\text { bre y final del concepto "solidaridad" por parte } \\
\text { de los alumnos. }\end{array}$ \\
\hline \multirow{3}{*}{ 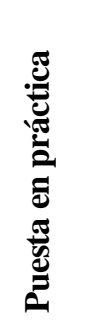 } & \multirow{3}{*}{$\begin{array}{l}\text { - Poner en práctica las sesiones planifi- } \\
\text { cadas. }\end{array}$} & $\begin{array}{l}\text { - Selección de los días de realización a partir de } \\
\text { la disponibilidad ofrecida por la maestra del cen- } \\
\text { tro. }\end{array}$ \\
\hline & & $\begin{array}{l}\text { - Preparación y disposición del aula y recursos } \\
\text { materiales dependiendo de las actividades que se } \\
\text { vayan a desarrollar. }\end{array}$ \\
\hline & & - Desarrollo de las sesiones y recogida de datos. \\
\hline \multirow{4}{*}{ 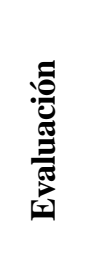 } & \multirow{4}{*}{$\begin{array}{l}\text { - Conocer los resultados de la puesta en } \\
\text { práctica de las sesiones. } \\
\text { - Evaluar niveles de consecución de los } \\
\text { objetivos propuestos. } \\
\text { - Realizar propuestas de mejora. }\end{array}$} & - Análisis de los resultados obtenidos. \\
\hline & & $\begin{array}{l}\text { - Valoración de las variables que puedan haber } \\
\text { afectado a los resultados. }\end{array}$ \\
\hline & & - Realización de un informe de resultados, con- \\
\hline & & clusiones y propuestas de mejora. \\
\hline
\end{tabular}

\section{5.- RESULTADOS}

\section{Cuestionario inicial}

Según los resultados obtenidos, el $95.7 \%$ del alumnado conoce a alguien de su propia clase que necesita algún tipo de ayuda y solo un $5 \%$ no conoce a alguien que se encuentre en esa situación (tabla 2). Igualmente, el porcentaje es muy elevado cuando se pregunta a los alumnos y alumnas si se sienten bien cuando hacen algo por los demás y solo uno de ellos (4.3\%) afirma no colaborar en las tareas de casa.

El 60.9\% no conocía al inicio del proyecto el significado de las siglas ONG. Sin embargo, todos coinciden en que es positivo que existan voluntarios para ayudar a otras personas.

Cuando el nivel de preguntas desciende a su entorno próximo, solo dos tercios de la clase conocen a alguien en su barrio a los que podrían ayudar. Por el contrario, solo un niño afirma no poder ayudar a los demás. Así mismo, únicamente un alumno admite que no lo gusta hacer favores a los demás y exige algo a cambio cuando los hace.

El 100\% del alumnado entiende que una persona solidaria es altruista y ayuda a los demás sin esperar nada a cambio. Igualmente, cuando planteamos situaciones relacionadas con el aula de música, el $100 \%$ del alumnado compartiría su libro o material de música con su compañero. Pero cuando se trata de coger de la mano a un compañero durante una danza, el número de negativas aumenta considerablemente y supera el $20 \%$. 
Tabla 2. Porcentajes cuestionario inicial

\begin{tabular}{lcc}
\hline \multicolumn{1}{c}{ Cuestiones } & Sí (\%) & NO (\%) \\
\hline 1. ¿Conoces a alguien de tu clase que necesite ayuda? & 95.7 & 4.3 \\
2. ¿Te sientes bien cuando haces algo por los demás? & 100.0 & 0.0 \\
3. ¿Colaboras con las tareas de casa? & 95.7 & 4.3 \\
4. ¿Sabes lo que es una ONG? & 39.1 & 60.9 \\
5. ¿Te parece bien que existan voluntarios que ayuden a otras personas? & 100.0 & 0.0 \\
6. ¿Conoces a alguien en tu barrio que requiera tu ayuda o que pienses que lo & 60.9 & 39.1 \\
puedes ayudar? & 95.7 & 4.3 \\
7. ¿Crees que puedes ayudar a los demás? & 4.3 & 95.7 \\
8. No me gusta hacer favores a los demás & 100.0 & 0.0 \\
9. Una persona solidaria es generosa y altruista & 100.0 & 0.0 \\
10. Comparto el material con mi compañero & 78.3 & 21.7 \\
11.En una danza evito coger la mano de algún que otro compañero & \\
\hline
\end{tabular}
$N=23$

Por último, ante los diferentes supuestos planteados al alumnado (tabla 3), más del 90\% distingue entre acciones solidarias y no solidarias.

Tabla 3. Porcentajes cuestionario inicial (reconocimiento de situaciones solidarias)

\begin{tabular}{lcc}
\hline \multicolumn{1}{c}{ SUPUESTOS } & $\begin{array}{c}\text { Verdadero } \\
\text { (\%) }\end{array}$ & $\begin{array}{c}\text { Falso } \\
\text { (\%) }\end{array}$ \\
\hline $\begin{array}{l}\text { 1. Una acción solidaria sería la siguiente: Juan no ha podido ir a clase porque es- } \\
\text { taba enfermo. Marta se ofrece para ayudarle con los deberes. }\end{array}$ & 87.0 & 13.0 \\
$\begin{array}{l}\text { 2. Una acción no solidaria sería la siguiente: unos bomberos ayudan a salir de un } \\
\text { incendio a Pablo y su gato, a pesar de que la situación era de alta peligrosidad. }\end{array}$ & 13.0 & 87.0 \\
$\begin{array}{l}\text { 3. Una acción solidaria sería la siguiente: Laura se ha hecho un esguince en el } \\
\text { tobillo y no puede ensayar la danza para fin de curso. Sus compañeros deciden } \\
\text { dejarla en clase y no bajarla al salón de actos para que no moleste. }\end{array}$ & 0.0 & 100.0 \\
$\begin{array}{l}\text { 4. Una acción solidaria sería la siguiente: la madre de Luis ha olvidado ponerle el } \\
\text { almuerzo en la mochila y Antonio decide compartir su bocadillo con él. }\end{array}$ & 95.7 & 4.3 \\
\hline$N=23$ & &
\end{tabular}

Los resultados obtenidos de este cuestionario inicial permitieron tener una idea aproximada de cómo entiende y evidencia nuestro alumnado el concepto de solidaridad a través de acciones cotidianas próximas a él. Esta información orientó considerablemente la actuación docente y favoreció la adaptación de las actividades al nivel del grupo-clase.

\section{Cuestionario de evaluación}

A continuación se presenta un resumen de los resultados obtenidos del cuestionario de evaluación. Una vez puesta en práctica la unidad didáctica, el 95.7\% concibe el valor de la solidaridad como un sentimiento de afecto que implica participación activa y ayuda a aquellos que la necesitan (tabla 4). Todo el grupo-clase entiende el concepto de voluntariado como una ayuda que se presta a otros sin pedir nada a cambio. También reconocen un ejemplo de acto solidario y saben lo que es un concierto benéfico o una ONG.

Más del 90\% del alumnado, a la hora de actuar, recuerda los consejos que se han dado en clase, considera interesante reflexionar en el aula de música sobre valores como la solidaridad y entiende mejor el concepto de este valor una vez trabajado a lo largo de este proyecto (tabla 5). 
Tabla 4. Porcentajes cuestionario de evaluación

\begin{tabular}{lcc}
\hline \multicolumn{1}{c}{ Cuestiones } & Sí (\%) & NO (\%) \\
\hline $\begin{array}{l}\text { La solidaridad es un sentimiento de afecto que implica par- } \\
\text { ticipación activa y ayuda }\end{array}$ & 95.7 & 4.3 \\
$\begin{array}{l}\text { El voluntariado es una ayuda que se presta a otros sin pedir } \\
\text { nada a cambio }\end{array}$ & 100.0 & 0.0 \\
¿Donar sangre en un acto solidario? & 100.0 & 0.0 \\
Sé lo que es un concierto benéfico & 100.0 & 0.0 \\
Sé lo que es una ONG & 100.0 & 0.0 \\
\hline$N=23$ & &
\end{tabular}

Tabla 5. Porcentajes cuestionario de evaluación (consideraciones y opiniones del alumnado)

\begin{tabular}{lcc}
\hline \multicolumn{1}{c}{ Cuestiones } & SÍ (\%) & NO (\%) \\
\hline Recuerda consejos que se han dado en clase & 91.3 & 8.7 \\
Considera interesante reflexionar sobre la solidaridad & 100.0 & 0.0 \\
$\begin{array}{l}\text { Entiende mejor qué es la solidaridad después de las sesio- } \\
\text { nes realizadas }\end{array}$ & 100.0 & 0.0 \\
\hline
\end{tabular}
$N=23$

Por último, con respecto a las actividades que más han gustado al alumnado (figura 4), las más valoradas han sido el blog, el disco-fórum y las propuestas solidarias. Las que ocupan las últimas posiciones son la actividad de flauta, la lluvia de ideas y la realización de frases solidarias. Podemos entender que las actividades que se han propuesto a través de las TICs (presentaciones visuales, vídeos, etc.) han sido las más votadas por el grupo. La actividad del blog En búsqueda de la solidaridad ocupa la primera posición, quizá porque aparecían personajes más afines al alumnado que tienen significación e interés para ellos (Sergio Ramos, Shakira...). Además, los enlaces que aparecen en el blog han captado muy bien la atención del alumnado, han escuchado atentos sus canciones y han entendido el significado de la solidaridad a través de la música.

\section{Evaluación}

La evaluación de los valores y actitudes adquiridos presenta limitaciones desde el punto de vista

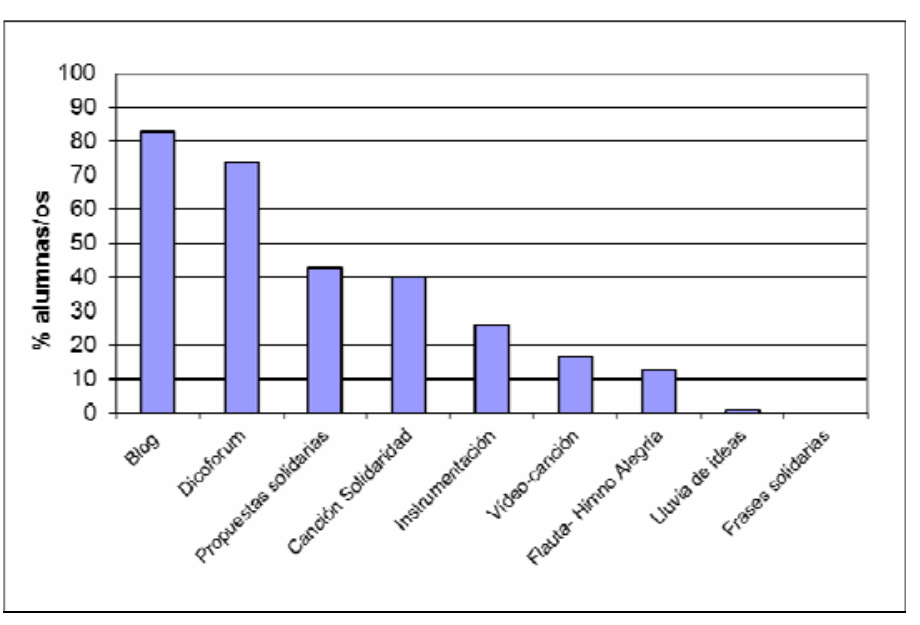

Figura 4. Actividades que más han gustado al alumnado temporal, pues son cambios que se producen a largo plazo, y desde el punto de vista del control de variables que puedan intervenir en los resultados finales. En nuestro caso concreto, las intervenciones educativas desde la familia, los mass media y los espacios no escolares escapan del ámbito de acción de nuestro proyecto. Sin embargo, es posible constatar mediante los instrumentos de evaluación seleccionados una variable muy importante: verificar si las actividades, tareas y actuación del docente, en su conjunto, han contribuido a la adquisición de conductas relacionadas con el valor de la solidaridad y, consecuentemente, si los objetivos propuestos se han alcanzado. 
Los resultados muestran que la mayoría del grupo-clase ha acogido la iniciativa con gran interés y motivación. El alumnado ha reflexionado sobre el valor de la solidaridad y ha conocido estrategias o formas de ser solidario desde sus posibilidades, en el aula de educación musical, en su entorno próximo y en la realidad social, con ejemplos prácticos que han generado una perspectiva cercana y una implicación efectiva.

Por último, el alumnado ha mostrado interés por las actividades programadas, ha reflexionado sobre el valor de la solidaridad en su vida cotidiana y se ha planteado el concepto de un valor cuya proyección es totalmente necesaria en las edades en las que está comprendida la etapa de Educación Primaria.

\section{...Coda}

Hemos constatado que el área de Música es un medio idóneo para trabajar los diferentes valores con los educandos (diálogo, respeto, igualdad, solidaridad, tolerancia, etc.). Así mismo, consideramos que la adquisición de pautas de comportamiento o hábitos relacionados con un determinado valor, en este caso la solidaridad, es posible tras la aplicación de un "programa" educativo. Coincidimos con Fernández (2003) cuando afirma que la música es un vehículo para trabajar la educación en valores y la reflexión sobre los estereotipos sociales, así como una alternativa para favorecer la transformación del rol de la música en la escuela.

A través de nuestra propuesta didáctica hemos conseguido interrelacionar la educación en valores con la educación musical de una forma cohesionada, pues entendemos que tienen que existir nexos de unión entre las distintas disciplinas y la educación en valores. Éstos deben comprenderse y trabajarse de forma global y continuada. La adquisición de valores necesita del trabajo conjunto de todo el profesorado y de la participación de la comunidad educativa al completo. Deseamos que esta experiencia sea el punto de partida de otras a mayor escala a partir de la intervención y cooperación entre el profesorado de distintas áreas y los diferentes agentes educativos.

Finalmente, nos gustaría agradecer al CEIP Los Álamos y, muy especialmente a la maestra de música, María Luisa López Orenes, su calurosa acogida y disponibilidad para que este proyecto se pudiera llevar a la práctica.

\section{6.- REFERENCIAS BIBLIOGRÁFICAS}

Fernández, F. (2003). Música, currículo y educación en valores. Cuadernos de pedagogía, 328, 62-65.

Herrera, S. (2007). El aula de música al servicio de la educación en valores. Conocimiento, educación y valores. Disponible en http://acosoescolar.es/valores/Taller/Herrera_R_S-UVigo-taller.pdf [Consulta: 2011, 7 de agosto].

Ortega, P. y Mínguez, R. (2001). Los valores en la educación. Barcelona: Ariel.

Peñalver, J. M. (2010). La improvisación musical y su relación con la educación en valores. Justificación y elaboración de un diseño curricular básico para el área de música de la educación secundaria obligatoria. Quaderns digitals: Revista de Nuevas Tecnologías y Sociedad, 64, 1-10.

Tiburcio, E. (2010). Canciones y discusión de dilemas en el desarrollo de valores. Una experiencia de intervención en Escuelas Primarias de la República Dominicana. Lista Electrónica Europea de Música y Educación (LEEME), 25, 123-148.

Touriñán, J. M. (2009). La escuela entre la permanencia y el cambio. Revista de Ciencias de la Educación, 218, 127-149.

Vicente, G. (2007). Música y valores: diálogo, tolerancia y solidaridad en canciones infantiles. Música y educación, 71, 17-43.

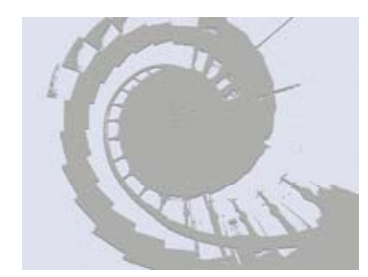

